\title{
Template Design and Analysis: Integrating Informatics Solutions to Improve Clinical Documentation
}

\author{
Justin lannello, DO, MBA; Nida Waheed, MD, MPH; and Patrick Neilan, DO
}

Background: Electronic health record templates have served a variety of functions, such as improving documentation for reliable reporting of health care outcomes. Standardizing template documentation has been important for accurately capturing case workload and supporting quality improvement initiatives.

Methods: North Florida/South Georgia Veterans Health System consists of 2 Florida-based hospitals: Malcom Randall Veterans Affairs Medical Center (MRVAMC) in Gainesville, and Lake City Veterans Affairs Medical Center (LCVAMC). In the first quarter of fiscal year 2017, MRVAMC and LCVAMC had a below-average case severity index (CSI) of 0.76 and 0.81 , respectively, compared with that of the 0.96 national average for the Veterans Health Administration
(VHA). An innovative history and physical template design with embedded informatics tools was created to improve clinical documentation.

Results: Compliance with standardized history and physical medicine template use was monitored for about 1 year after standardized template implementation. Compliance improved from $43.2 \%$ in June 2018 to $89.9 \%$ in June 2019 at MRVAMC and increased from $48.2 \%$ in June 2018 to $96.9 \%$ in June 2019 at LCVAMC. CSI improved to 0.97 at MRVAMC and 1.07 at LCVAMC in the first quarter of fiscal year 2019, which exceeded the VHA national average of 0.89 during the same period.

Conclusions: NF/SGVHS integrated informatics solutions within template design was associated with an increase in CSI via improved clinical documentation.
S tandardized template design is a useful tool to improve clinical documentation and reliable reporting of health care outcomes when constructed with clear objectives and with collaboration of key stakeholders. A standardized template should not only capture accurate diagnostic information, but also inform quality improvement (QI) measures and best practices.

Kang and colleagues showed that a correlation exists between organizational satisfaction and improved quality outcomes. ${ }^{1}$ A new initiative should have a well-defined purpose reinforced by collaborative workgroups and engaged employees who understand their clinical care role with electronic health record (EHR) modifications.

Several studies have shown how the usefulness of templates achieve multipurpose goals, such as accurate documentation and improved care. Valluru and colleagues showed a significant increase in vaccination rates for patients with inflammatory bowel disease after implementing a standardized template. ${ }^{2}$ By using a standardized template, Thaker and colleagues showed improved documentation regarding obesity and increased nutritional and physical activity counseling. ${ }^{3}$ Furthermore, Grogan and colleagues showed that templates are useful for house staff education on International Classification of Diseases (ICD) terminology and demonstrated improved documentation in the postintervention group. 4,5

This article discusses the US Department of Veterans Affairs (VA) North Florida/South Georgia Veterans Health System (NF/SGVHS) integrated informatics solutions within template design in the Veterans Health Administration (VHA) EHR system that was associated with an increase in its case severity index (CSI) through improved clinical documentation capture.

\section{METHODS}

According to policy activities that constitute research at NF/SGVHS, institutional review board approval was not required as this work met the criteria for operational improvement activities exempt from ethics review.

NF/SGVHS includes 2 hospitals: Malcom Randall VA Medical Center (MRVAMC) in Gainesville, Florida, and Lake City VA Medical Center (LCVAMC) in Lake City, Florida. MRVAMC is a large, la, academic VA facility composed of rotating residents and fellows and includes multiple specialty care services. LCVAMC is a smaller, nonteaching facility.

\section{Template Design Impact}

CSI is a risk-adjusted formula developed by the Inpatient Evaluation Center within VHA. CSI is incorporated into the VHA quality metrics reporting system,
Author affiliations can be found at the end of the article.

Correspondence: Justin lannello (jliannello22@gmail.com)

Fed Pract. 2020;37(11):527-531. doi:10.12788/fp.0072 
TABLE Case Severity Index By Location

\begin{tabular}{lcc} 
Locations & $\begin{array}{c}\text { Preimplementation } \\
\text { (FY 2017, Q1) }\end{array}$ & $\begin{array}{c}\text { Postimplementtion } \\
\text { (FY 2019, Q1) }\end{array}$ \\
\hline VHA & 0.96 & 0.89 \\
\hline MRVAMC & 0.76 & 0.97 \\
\hline LCVAMC & 0.81 & 1.07
\end{tabular}

Abbreviations: FY, fiscal year; LCVAMC, Lake City Veterans Affairs Medical Center; MRVAMC, Malcom Randall Veterans Affairs Medical Center; Q, quarter; VHA, Veterans Health Administration.

\section{FIGURE 1 H\&P Medicine Template Primary Reason(s) for Admission and Chronic Comorbitities, NF/SGVHS, Example}

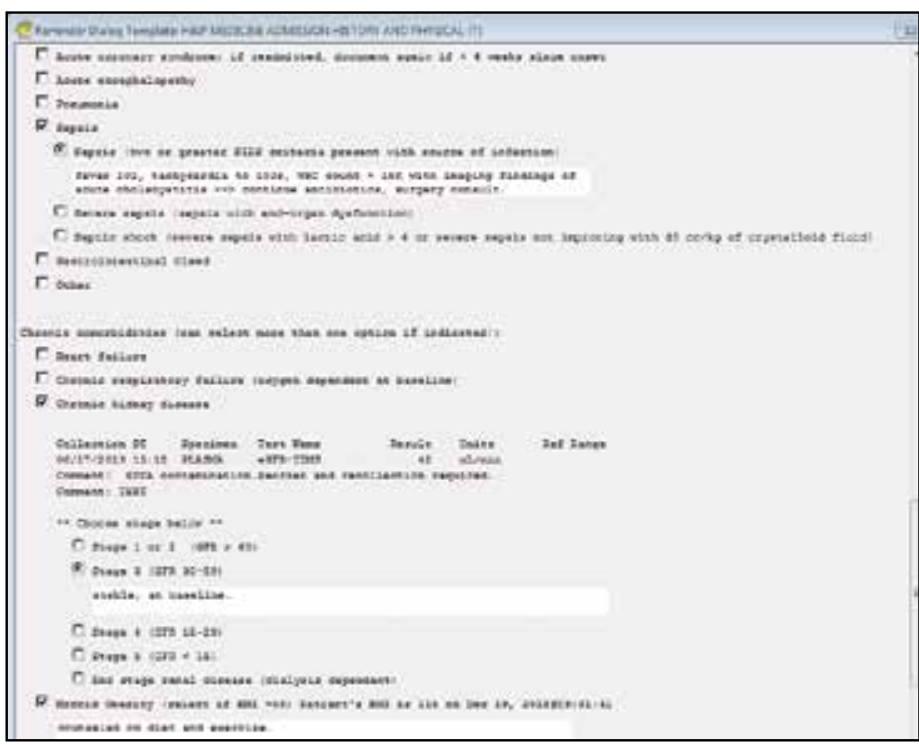

Abbreviations: H\&P, history and physical; NF/SGVHS, North Florida/South Georgia Veterans Health System.

Strategic Analytics for Improvement and Learning (SAIL). CSI risk-adjusts metrics such as length of stay and mortality before releasing SAIL reports. CSI is calculated separately for acute level of care (LOC) and for the intensive care unit (ICU). In fiscal year (FY) 2017, acute LOC preimplementation data for CSI at NF/SGVHS were 0.76 for MRVAMC and 0.81 for LCVAMC, which was significantly below the national VHA average of 0.96 (Table).

A below-average CSI conveys a less complicated case mix compared with most other VA facilities. Although smaller VA facilities may have a less complicated case mix, it is unusual for large, tertiary care la VA facilities to have a low CSI. This low CSI is usually due to inadequate documentation, which affects not only risk-adjusted quality metrics outcomes, but also potential reimbursement. ${ }^{6}$

An interdisciplinary team composed of attendings, residents, and a clinical document improvement specialist identified the belowaverage acute LOC CSI for MRVAMC and LCVAMC compared with that of the national VHA average. Further analysis by chart reviews showed inconsistencies with standardized documentation despite prior health care provider education on ICD terminology and specific groups of common comorbidities analyzed in administrative data reviews for riskadjustment purposes, known as Elixhauser comorbidities. ${ }^{5,7}$

A chart review showed lack of clarity regarding primary reason(s) for admission and chronic comorbidities within NF/SGVHS. Using Pareto chart analysis, the template team designed a standardized history and physical $(\mathrm{H} \& \mathrm{P})$ medicine template based on NF/SGVHS common medicine admissions (Figure 1). A Pareto chart is a valuable QI tool that assists with identifying majority contributors to a problem(s) being analyzed when evaluating a large set of data points. Subsequently, this tool helps focus direction on QI efforts. ${ }^{8}$

The template had the usual H\&P elements not shown (eg, chief complaint, history of present illness, etc), and highlights the assessment/plan section containing primary reason(s) for admission and chronic comorbidities (Figure 1). The complete assessment and plan section on the template can be found in the Appendix.

To simplify the template interface, only single clicks were required to expand diagnostic and chronic comorbidity checkboxes. Subcategories then appeared to select diagnosis and chronic comorbidities along with free text for additional documentation.

In addition, data objects were created within the template that permitted the ability to retrieve information from the VHA EHR and insert specific data points of interest in the template; for example, body mass index to assess degree of obesity and estimated glomerular filtration rate to determine the stage of chronic kidney disease. This allowed users to easily reference data in one template in lieu of searching for data in multiple places in the EHR. ${ }^{9}$ 


\section{RESULTS}

The standardized H\&P medicine template was implemented at MRVAMC and LCVAMC in June 2018 (the final month of the third quarter of FY 2018). As clinical providers throughout NF/SGVHS used the standardized template, acute LOC postimplementation data for CSI significantly improved. Although the national VHA average slightly decreased from 0.96 in the first quarter of FY 2017 to 0.89 , in the first quarter of FY 2019, MRVAMC acute LOC CSI improved from 0.76 to 0.97 , and LCVAMC acute LOC CSI improved from 0.81 to 1.07 during the same period.

In addition, compliance also was monitored within MRVAMC and LCVAMC for about 1 year after standardized H\&P medicine template implementation. Compliance was determined by how often the standardized $H \& P$ medicine template was used for inpatient medicine admissions to the acute care wards vs other H\&P notes used (such as personalized templates).

Methodology for compliance analysis included acquisition of completed H\&P medicine notes from June 18, 2018 to June 30, 2019 , within the VHA Veterans Information Systems and Technology Architecture (VistA) clinical and business information system using the search strings: "H\&P admission history and physical" and "history of present illness." 10

A review identified 10,845 completed medicine H\&P notes. Nine hundred eighteen notes were excluded as their search function yielded a location not corresponding to MRVAMC or LCVAMC. Of the 9,927 notes remaining, 8,025 of these were completed medicine H\&P notes at MRVAMC and 1,902 were completed medicine $H \& P$ notes at LCVAMC (Figure 2).

From June 18, 2018 to June 30, 2019 at MRVAMC, compliance was reviewed monthly for the 8,025 completed $H \& P$ medicine notes. Of the completed $H \& P$ medicine notes, the standardized H\&P medicine template was used $43.2 \%$ in June 2018. By June 2019, MRVAMC clinical providers demonstrated significant improvement for standardized $\mathrm{H} \& \mathrm{P}$ medicine template use at $89.9 \%$ (Figure 3 ). Total average compliance from June 18 , 2018 to June 30 , 2019, was $88.4 \%$, which
FIGURE 2 Standardized H\&P Medicine Template

Compliance Evaluation Study

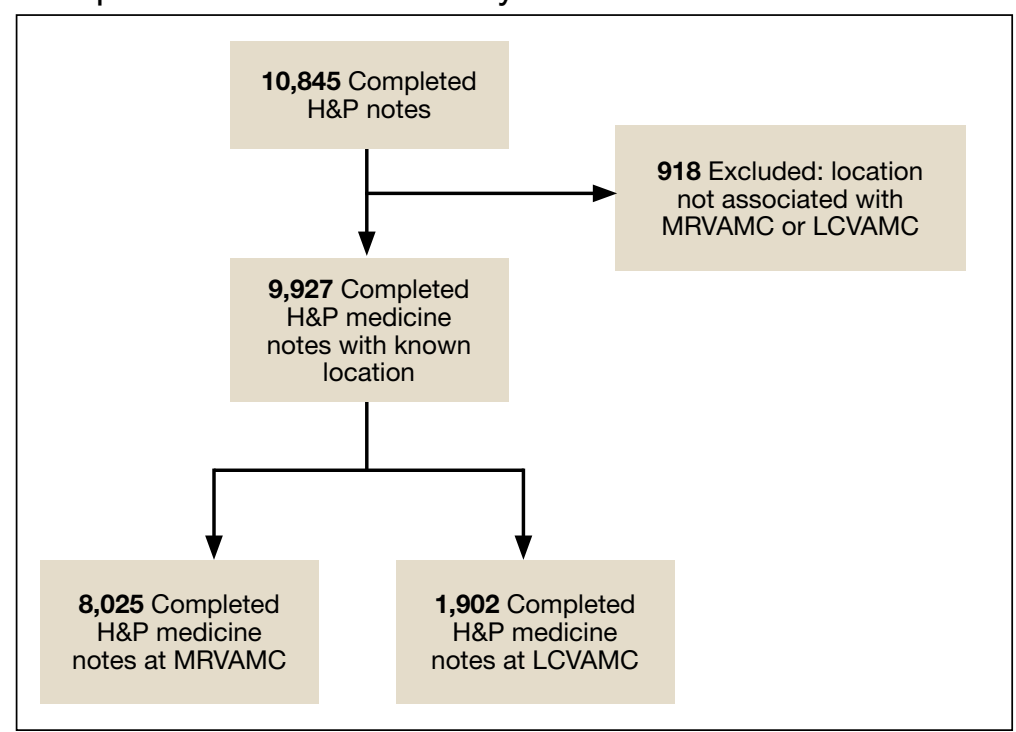

Abbreviations: H\&P, history and physical; LCVAMC, Lake City Veterans Affairs Medical Center; MRVAMC, Malcom Randall Veterans Affairs Medical Center.

doubled compliance from the initial introduction of the standardized H\&P medicine template.

Compliance was reviewed monthly for the 1,902 completed H\&P medicine notes from June 18, 2018 to June 30, 2019, at LCVAMC. Of the completed H\&P medicine notes, the standardized template was used $48.2 \%$ of the time in June 2018. By June 2019, LCVAMC clinical providers demonstrated significant improvement for standardized H\&P medicine template use, which increased to $96.9 \%$. Total average compliance from June 18, 2018 to June 30, 2019 , was $93.8 \%$, which was almost double the baseline compliance rate.

\section{DISCUSSION}

Template design with clear objectives, strategic collaboration, and integrated informatics solutions has the potential to increase accuracy of documentation. As shown, the NF/SGVHS template design was associated with significant improvement in acute LOC CSI for both MRVAMC and LCVAMC due to more accurate documentation using the standardized H\&P medicine template.

Numerous factors contributed to the success of this template design. First, a clear vision for application of the template was 


\section{FIGURE 3 Standardized History and Physical Medicine Template Monthly Compliance Data}

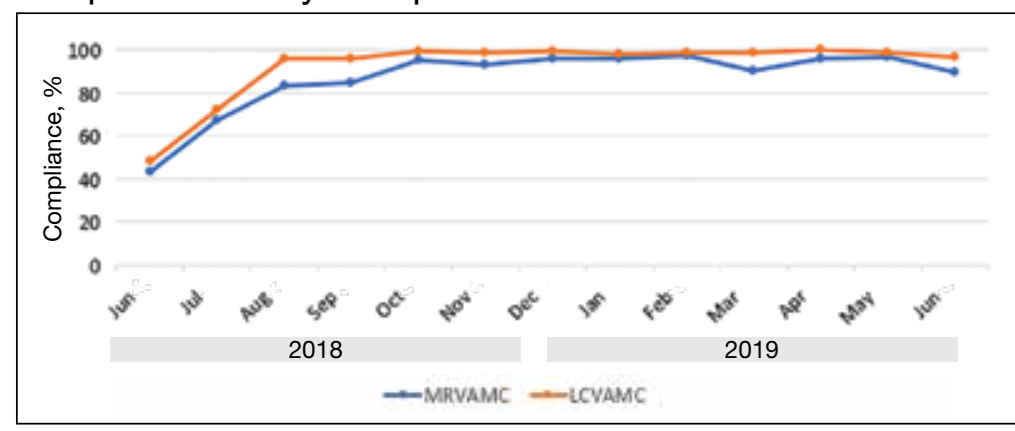

Abbreviations: LCVAMC, Lake City Veterans Affairs Medical Center; MRVAMC, Malcom Randall Veterans Affairs Medical Center.

communicated with key stakeholders. In addition, the template design team was focused on specific goals rather than a one size fits all approach, which was crucial for sustainable execution. Although interdisciplinary teamwork has the potential to result in innovative practices, large multidisciplinary teams also may have difficulty establishing a shared vision that can result in barriers to achieving project goals.

Balancing standardization and customization was essential for user buy-in. As noted by Gardner and Pearce, inviting clinical providers to participate in template design and allowing for customization has the potential to increase acceptance and use of templates. ${ }^{11}$ Although the original design for the standardized H\&P medicine template started with the medicine service at NF/SGVHS, the design framework is applicable to numerous services where various clinical care elements can be customized.

Explaining the informatics tools built into the template allowed clinicians to see opportunities to improve clinical documentation and the impact it has on reporting health care outcomes. When improvement work involves integrating clinical care delivery and administrative expectations, it is essential that health care systems understand and strategically execute project initiatives at this critical juncture.

Finally, incorporation of a sustainability plan when process improvement strategies are implemented is vital. In addition to collaboration with the clinical providers during design and implementation of the standardized template, leadership buy-in was key.
Compliance with standardized H\&P medicine template use was monitored monthly and reviewed by the NF/SGVHS Chief of Staff.

As noted, LCVAMC postimplementation acute LOC CSI was higher than that of MRVAMC despite being a smaller facility. This might be due to the MRVAMC designation as a teaching institution. Medicine is the only inpatient service at LCVAMC staffed by hospitalists with limited specialists available for consultation, whereas MRVAMC is a tertiary care teaching facility with numerous inpatient services and subspecialties. As LCVAMC has more continuity, house staff rotating at MRVAMC require continued training/education on new templates and process changes.

\section{Limitations}

Although standardized template design was successful at NF/SGVHS, limitations should be noted. Our clinical documentation improvement (CDI) program also was expanded about the same time as the new templates were released. The expansion of the CDI program in addition to new template design likely had a synergistic effect on acute LOC CSI.

CSI is a complex, risk-adjusted model that includes numerous factors, including but not limited to diagnosis and comorbid conditions. Other factors include age, marital status, procedures, source of admission, specific laboratory values, medical or surgical diagnosis-related group, intensive care unit stays, and immunosuppressive status. CSI also includes operative and nonoperative components that average into an overall CSI. As the majority of CSI is composed of nonoperative constituents within NF/SGVHS, we do not believe this had any substantial impact on reporting of CSI improvements.

In addition, template entry into VHA EHR requires a location selection (such as a clinic name or ward name following an inpatient admission). Of the 10,845 completed H\&P medicine notes identified in VistA, 918 notes were excluded from analysis as their search function yielded a location not corresponding to MRVAMC or LCVAMC. For the 918 notes excluded, we believe this was likely due to user error where locations not related to MRVAMC or LCVAMC were 
selected during standardized H\&P medicine template entry.

\section{CONCLUSIONS}

After the NF/SGVHS implementation of a uniquely designed template embedded with informatics solutions within the VHA EHR, the CSI increased due to more accurate documentation.

Next steps include determining the impact of the NF/SGVHS template design on potential reimbursement and expanding template design into the outpatient setting where there are additional opportunities to improve clinical documentation and reliable reporting of health care outcomes.

\section{Acknowledgments}

The authors thank the following individuals for their experience and contribution: Beverley White is the Clinical Documentation Improvement Coordinator at North Florida/South Georgia Veterans Health System and provided expertise on documentation requirements. Russell Jacobitz and Susan Rozelle provided technical expertise on electronic health record system enhancements and implemented the template design. Jess Delaune, MD, and Robert Carroll, MD, provided additional physician input during template design. We also acknowledge the Inpatient Evaluation Center (IPEC) within the Veterans Health Administration (VHA). IPEC developed the case severity index, a risk-adjusted formula incorporated into the VHA quality metric reporting system, Strategic Analytics for Improvement and Learning (SAIL).

\section{Author affiliations}

Justin lannello is National Lead Physician Utilization Management Advisor for the Veterans Health Administration and Associate Chief of Staff for Clinical Informatics at the Southeast Louisiana Veterans Health Care System in New Orleans. Nida Waheed is Chief Resident in Quality and Patient Safety for the Department of Internal Medicine, and Patrick Neilan is Chief Resident for the Department of Internal Medicine, both at University of Florida in Gainesville.

\section{Author disclosures}

The authors report no actual or potential conflicts of interest with regard to this article.

\section{Disclaimer}

The opinions expressed herein are those of the authors and do not necessarily reflect those of Federal Practitioner, Frontline Medical Communications Inc., the US Government, or any of its agencies.

\section{References}

1. Kang R, Kunkel S, Columbo J, et al. Association of Hospital Employee satisfaction with patient safety and satisfaction within Veterans Affairs Medical Centers. Am J Med. 2019;132(4):530-534.e1. doi: 10.1016/j.amjmed.2018.11.031

\section{APPENDIX NF/SGVHS Assessment/Plan on the Standardized H\&P Medicine Template}

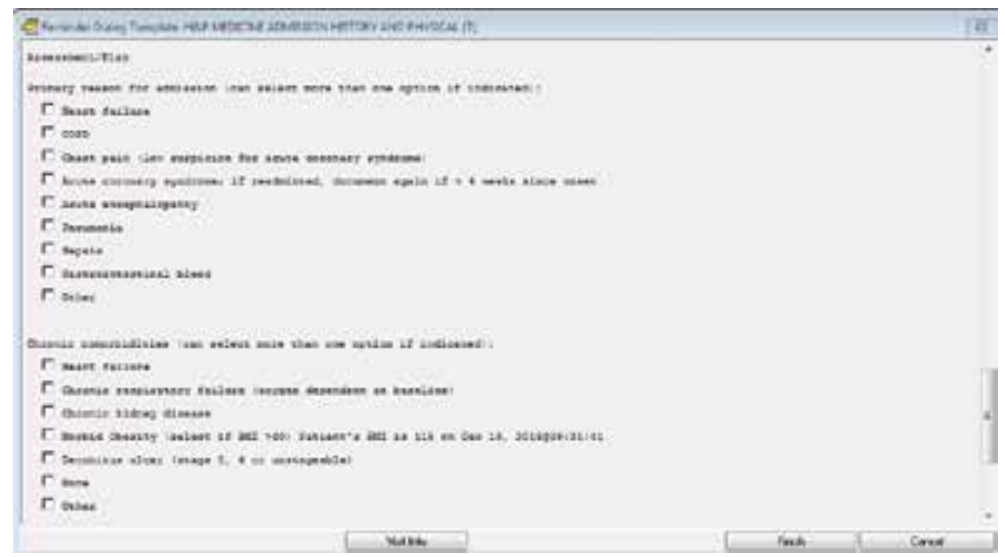

Abbreviations: H\&P, history and physical; NF/SGVHS, North Florida/South Georgia Veterans Health System.

2. Valluru, N, Kang L, Gaidos JK. Health maintenance documentation improves for veterans with IBD using a template in the Computerized Patient Record System. Dig Dis Sci. 2018;63(7):1782-1786. doi:10.1007\%2Fs10620-018-5093-5

3. Thaker VV, Lee F, Bottino CJ, et al. Impact of an electronic template on documentation of obesity in a primary care clinic. Clin Pediatr. 2016;55(12):1152-1159. doi:10.1177/0009922815621331

4. Grogan EL, Speroff T, Deppen S, et al. Improving documentation of patient acuity level using a progress note template. J Am Coll Surg. 2004;199(3):468-475. doi:10.1016/j.jamcollsurg.2004.05.254

5. Centers for Disease Control and Prevention. Classification of diseases, functioning, and disability. https://www .cdc.gov/nchs/icd/index.htm. Updated June 30, 2020. Accessed October 12, 2020.

6. Marill K A, Gauharou ES, Nelson BK, et al. Prospective, randomized trial of template-assisted versus undirected written recording of physician records in the emergency department. Ann Emerg Med. 1999;33(5):500509. doi:10.1016/S0196-0644(99)70336-7

7. Elixhauser A, Steiner C, Harris DR, et al. Comorbidity measures for use with administrative data. Med Care. 1998;36(1):8-27. doi:10.1097/00005650-199801000-00004

8. Hart KA, Steinfeldt BA, Braun RD. Formulation and applications of a probalistic Pareto chart. AIAA. 2015;0804. doi:10.2514/6.2015-0804

9. IBM. IBM knowledge center: overview of data objects. https://www.ibm.com/support/knowledgecenter /en/SSLTBW_2.3.0/com.ibm.zos.v2r3.cbclx01/data objects.htm. Accessed October 12, 2020.

10. US Department of Veterans Affairs. History of IT at VA. https://www.oit.va.gov/about/history.cfm. Accessed October 18, 2020.

11. Gardner CL, Pearce PF. Customization of electronic medical record templates to improve end-user satisfaction. Comput Inform Nurs. 2013;31(3):115-121. doi:10.1097/NXN.0b013e3182771814 